\title{
VARIACIONES EN LAS PAUTAS DE LOCALIZACION INDUSTRIAL: FORMACION DEL "CORREDOR INDUSTRIAL DEL EBRO" Y GENERAL MOTORS
}

\author{
María Pilar ALONSO LOGROÑO ${ }^{1}$
}

Universidad de Zaragoza

\begin{abstract}
RESUMEN: El trabajo examina como la ubicación de la factoría de General Motors E., a treinta kilómetros del casco urbano de Zaragoza, está contribuyendo a la acentuación de dos aspectos: la implantación de un nuevo modelo de localización industrial cada vez más alejado de la ciudad, en la medida que los medios de transporte se van desarrollando y la consolidación del "Corredor Industrial del Ebro", como una de las zonas más dinámicas de todo el "Eje del Ebro".
\end{abstract}

\begin{abstract}
The work revises in what manners the location of the factory General Motors E., 30 kilometres far from the city centre of Zaragoza, is helping to the remalk of two aspects: the research of a new industrial setting far from the city this due to the new and more advanced means of transport and on the other hand the consolidatione of "Corredor Industrial del Ebro" live one of the most dinamic and working areas into the Ebro net work.
\end{abstract}

Sumario: Introducción.- Localizaciones industriales en la Primera Revolución Industrial.Localizaciones industriales con el Polo de Desarrollo y los Poligonos Industriales.- Implantación de General Motors y los cambios en el modelo de localización industrial.- Consolidación del "Corredor Industrial del Ebro".- Razones de Crecimiento del Corredor Industrial del Ebro".- Conclusiones.Bibliografia.

\section{INTRODUCCION}

El denominado "Corredor Industrial del Ebro", constituye hoy un espacio bastante bien delimitado dentro de la provincia de Zaragoza, compuesto por una serie de núcleos situados en la ribera de este río, con epicentro en Zaragoza y cuya área de atracción se extiende también por la ribera del Jalón.

\footnotetext{
1 Becaria de investigación del Dpto. de Geografía y Ordenación del Territorio.
} 
Este espacio se ha convertido en uno de los tramos más dinámicos del "Eje del Ebro", beneficiándose de las múltiples ventajas asociadas al global de este territorio, tan estratégico en la economía nacional y que además enlaza fácilmente con los principales ejes económicos europeos. De todo el eje del Ebro es en el tramo que hoy se delimita como el "Corredor Industrial del Ebro" donde esas ventajas de situación parecen concentrarse con mayor fuerza durante los últimos años.

Son varios los estudios que se han llevado a cabo sobre este "Corredor", comprobando que las tierras englobadas bajo esta denominación presentan condiciones muy similares para el desarrollo industrial que ya se ha comenzado a potenciar en él.

La consolidación de este Corredor es un hecho bastante reciente y relacionado estrechamente con la localización de la factoría de General Motors en Figueruelas, a comienzos de los ochenta, ya que hasta ese momento su expansión se limitaba a la periferia de la ciudad de Zaragoza y a algunos pequeños enclaves aislados.

Esta multinacional automovilística está marcando un nuevo modelo de localización industrial, en donde las distancias kilométricas para el desplazamiento, en especial las de los obreros, cada vez importan menos, aunque surgen otra serie de requisitos básicos que buscan las empresas a la hora de instalarse (situación geográfica, infraestructuras,...).

En este artículo analizaremos someramente los orígenes y la consolidación de este Corredor, desde los primeros momentos en que llegó la industrialización a la provincia, con una localización puntual unida al entramado urbano de Zaragoza, momento que puede considersarse como el origen de este corredor industrial, después su posterior expansión hacia la periferia y por último la superación del espacio ligado a la ciudad coincidiendo con la implantación de General Motors. En definitiva, se examina la variación producida en el modelo de localización de las industrias a medida que ha pasado el tiempo ${ }^{2}$.

2 Los dos primeros modelos de localización industrial constituyen dos etapas históricas que han sido estudiadas en diferentes trabajos sobre la industria aragonesa y a los que hemos acudido para realizar nuestra investigación. Sin embargo hemos creido conveniente hacer una pequeña recapitulación de los puntos más significativos para reflejar con más claridad la variación producida en los ultimos años en relación con los anteriores modelos de localizacion industrial.

De la bibliografía consultada destacar los siguientes trabajos:

CAMARA DE COMERCIO E INDUSTRIA DE ZARAGOZA (1978): "Estructura y Localización de la industria en Zaragoza". Tomo II, Zaragoza. 
Para ello se ha dividido el proceso en tres fases:

1- La relacionada con la Primera Revolución Industrial en la ciudad, de localización intraurbana.

2- La fase de creación del Polo de Desarrollo y de los distintos polígonos industriales, con una expansión hacia la periferia más cercana a la ciudad.

3- La implantación de General Motors, rebasando y extendiendo las localizaciones industriales a través de los ejes de entrada a la ciudad.

\section{LOCALIZACIONES INDUSTRIALES INTRAURBANAS EN LA PRIMERA REVOLUCION INDUSTRIAL.}

Para el análisis de las primeras localizaciones industriales tenemos que remontarnos a finales del siglo XIX, ya que lo que existía antes de esta fecha no eran más que pequeños talleres de tipo artesanal, muy relacionados en sus ubicaciones con el concepto medieval de gremios. A finales del siglo pasado es cuando comienza dentro del entramado urbano de Zaragoza un auténtico despegue industrial, aunque en aquellos momentos no presentaba demasiadas ventajas para las instalaciones fabriles $^{3}$, se vio favorecido principalmente por:

a) la situación de encrucijada entre otras regiones económicamente más fuertes,

b) la posibilidad de adquirir fácilmente recursos energéticos, por las buenas comunicaciones con centros que los poseían,

c) la instalación, en esas fechas, de las primeras líneas del ferrocarril nacional que llegan a la ciudad

CAMARA DE COMERCIO E INDUSTRIA DE ZARAGOZA (1982): " Los polígonos industriale en Aragón". C.A.Z.A.R., Zaragoza.

FAUS PUJOL, C. (1978): "El ferrocarril y la evolución urbana de Zaragoza". Geographicalia, Dpto. de Geografía, Universidad de Zaragoza. № 2.

MENSUA FERNANDEZ, S. (1954): "La localización de las industrias en Zaragoza". Geographicalia. Dpto de Geografía, Universidad de Zaragoza, año $1^{\circ}$, no $2,3, y 4$.

NAVARRO FERRER, A.M. (1962): " Geografía urbana de Zaragoza". Geographicalia IX. Dpto. de Geografía Aplicada del Instituto Elcano. C.S.I.C. Zaragoza.

SANCHO MARTI, J. (1989): "El espacio periurbano de Zaragoza". Ayuntamiento de Zaragoza, Servicio de acción cultural.

${ }^{3}$ Era una provincia eminentemente agrícola y ganadera, con pocas materias primas propias y con muy pocas iniciativas empresariales 
Zaragoza era sólo una "zona de paso" entre otras regiones españolas pioneras en su industrialización, pero comenzó a beneficiarse de su buena situación en el centro del fértil cuadrante del Noreste peninsular.

Se inicia el aprovechamiento de materias primas autóctonas: yesos, sal, arcilla,etc., de algunos cultivos industriales: algodón, remolacha, etc.. Se pone en funcionamiento la primera fábrica de gas en 1864 y la electricidad llega a Zaragoza en 1883. Con la presencia de ambas fuentes de energía en la ciudad se logra la instalación de empresas industriales dentro del perímetro urbano.

En 1861 se inaugura el ferrocarril que unía a Zaragoza con Barcelona, éste llegaba a una estación en la margen izquierda del Ebro: la estación del Arrabal. En 1864 se inaugura también el ferrocarril que enlazaba con Madrid que terminaba en la actual estación del Portillo, entonces denominada del "Campo Sepulcro". Las dos estaciones de ferrocarril creadas, una en cada margen del río Ebro, se unieron en 1870 a través del Puente de Hierro, ésto provocó la implantación de industrias a ambos lados de esta vía, así por ejemplo, la instalación de la "Industria Química de Zaragoza" (1898) estuyo relacionada con esta unión.

Numerosas industrias, especialmente las relacionadas con los transformados agrícolas empiezan a instalarse en Zaragoza. Las materias primas regionales les llegan desde distintos puntos a través del ferrocarril ${ }^{4}$. Entre todas ellas destaca la remolacha, muy abundante en la región desde finales del siglo pasado hasta los años sesenta, lo cual motivó el surgimiento de una gran cantidad de azucareras no sólo en la capital sino en toda la provincia ${ }^{5}$. Otros sectores que comienzan a aparecer entonces y a cobrar un gran renombre fueron el metalúrgico de transformados (cerrajes, tornillos,...), el de material eléctrico y el de las papeleras.

En esta primera etapa todas las ubicaciones industriales están unidas al entramado urbano y más concretamente al del ferrocarril, situándose un importante número de industrias a lo largo de sus vías, especialmente en la margen izquierda del Ebro, donde tenían un medio fácil de transporte para sus productos. También este tipo de localizaciones está ligado al área de residencia de la mano de

4 La red de ferrocarriles aunque no era muy densa, será en aquellos momentos un factor de atracción industrial muy importante.

5 Aragón llegó a convertir en el primer productor nacional de remolacha. 
obra que trabaja en ellas, al no estar los medios de transporte demasiado desarrollados, las industrias han de buscar para su instalación una zona cercana a donde residan sus obreros.

Zaragoza era una ciudad muy compacta, con una red radial de comunicaciones que partían desde el centro de la ciudad dirigiéndose hacia todas las direcciones, pero estos ejes todavía no se aprovecharán para las localizaciones industriales ya que aún se vive mucho de los propios abastecimientos locales y el transporte más desarrollado era el ferrocarril y no el de las carreteras.

De esta etapa quedan en el entramado de la ciudad residuos de pequeñas instalaciones industriales con pocos trabajadores y unos medios técnicos bastante atrasados. Aunque en esta primera fase de industrialización no se puede hablar todavía de un corredor industrial, puesto que era una localización muy puntual y ligada al casco urbano de Zaragoza, independientemente del tamaño de la empresa, podemos poner en ese momento el inicio para su posterior desarrollo a partir del entramado urbano.

\section{LOCALIZACIONES INDUSTRIALES CON EL POLO DE DESARROLLO Y LOS POLIGONOS INDUSTRIALES.}

Desde 1964, en que se conceden a la provincia de Zaragoza los privilegios de ser Polo de Desarrollo ${ }^{6}$, la situación descrita cambia. Entre los motivos por los que se elige a Zaragoza siguen primando su excelente situación geográfica, los importantes ejes de comunicación nacional que la atraviesan y la existencia de muchas pequeñas y medianas empresas con pocos recursos propios para su desarrollo.

Uno de los resultados más reseñables de esta acción es que la mayoría de las empresas que se acogen a los beneficios del Polo de Desarrollo ya existían antes, pero ubicadas en el interior del casco urbano. Se trata de aquellas empresas que se habían quedado anticuadas o necesitaban más espacio para sus instalaciones por lo que decidieron acogerse a esos beneficios, trasladándose hacia la

\footnotetext{
6 El Gobierno Español para paliar las diferencias que existían entre las distintas regiones españolas decide dar a unas cuantas zonas unos determinados privilegios económicos para que consigan igualarse con otras más desarrolladas.
} 
periferia de la ciudad donde existía más suelo y a precios más baratos. Sin embargo, pocas fueron las industrias de nueva creación durante esa época.

La nueva localización de las industrias que decicieron trasladarse coincidía con la de los principales ejes de salida de la ciudad, que con la mejora en los medios de transporte por carretera, se convertían en las zonas a las que más rápidamente se podía acceder y poseían todos los requisitos que las empresas buscaban.

Además de la concesión del Polo, a partir de 1975, se fueron aprobando la preparación de numerosos polígonos industriales, expandidos por toda la provincia, ya que la política del Polo parece que no había tenido demasiadas buenas repercusiones de cara a la expansión industrial de las pequeñas y medianas empresas. Tan sólo fueron las grandes las que en ese primer momento se trasladaron a la periferia inmediata de la ciudad: Pikolín, Puma Chauson, Edelvives, Nurel,...

Mediante estos polígonos se pretendía, que la mayoría de las pequeñas y medianas industrias, que todavía se asentaban en el interior del casco urbano, se trasladasen a ellos para poder ampliar y renovar sus instalaciones y que otras, de nueva creacción, fueran atraidas por nuevas infraestructuras.

La proliferación de polígonos fue muy numerosa, unos de creación estatal, otros municipales y otros privados. Sin embargo, en muchos de ellos la ocupación, en aquellos primeros momentos, fue muy escasa. Sobre los que mayor número de industrias se asentaron fueron aquellos que se localizaban en los ejes mejor comunicados de la ciudad, conformándose una ocupación industrial de forma radial respecto al casco urbano de Zaragoza y de distinta intensidad según los ejes.

La ciudad con estos hechos acaba rompiendo su estructura compacta, ensanchándose hacia los ejes de comunicación, no sólo a través de instalaciones industriales sino también de áreas residenciales. La imagen que a fines de los setenta presenta la ciudad en cuanto a instalaciones industriales es la siguiente:

- En el interior del casco urbano había todavía una importante presencia de pequeñas y medianas industrias que, a pesar de los problemas de falta de espacio, de contaminación, de atascos, en ese momento todavía no demasiado importantes, permanecen asentadas en el interior. 
- La carretera de Barcelona (Polígono de Malpica), tanto por el tipo de terrenos, poco fértiles para la agricultura, como por la buena situación en cuanto a accesos, era una de las mejores zonas para las ubicaciones industriales. Sin embargo, el mal estado de las infraestructuras hasta la creación de la autopista con Barcelona impidieron el desarrollo de este espacio. Las instalaciones que encontrábamos en aquellos primeros momentos estaban ubicadas de forma muy aislada.

- La carretera de Castellón fue ocupada muy pronto, por la excelente comunicación que tiene desde el centro urbano. El tipo de industria que se trasladó era de caracter pequeño y medio. En este eje, hay que destacar que, muchas de estas empresas se asentaron sobre espacios no declarados como industriales, por lo que restaron importantes zonas fértiles para la agricultura.

- En la carretera de Madrid no se conocieron durante esta fase más que unas localizaciones industriales muy dispersas, con industrias de mediano tamaño.

- La carretera de Logroño presentó una localización en ambas márgenes de la carretera pero con muy poca densidad, mezclándose con estas instalaciones industriales usos residenciales y agrícolas.

- En el resto de la provincia son muchos los municipios que establecieron en sus términos la creación de polígonos industriales locales, ofreciendo numerosas facilidades a las empresas que decidieran instalarse en ellos (donaciones de terrenos, exenciones fiscales,...). Sin embargo, fueron muy pocas las industrias que decidieron ubicarse fuera del área próxima a la ciudad, o si lo hicieron buscan núcleos de población importantes (Ejea, Tauste, Tarazona,...).

Un hecho común presente en la mayoría de los municipios donde se asientan estos polígonos industriales es que en pocos de ellos se tuvieron en cuenta el tipo de terreno que se elegía para la creacción del mismo, ésto tuvo como resultado que una gran cantidad de zonas muy fértiles desde el punto de vista agronómico, se calificaran como industriales, restando ese primer gran valor a los terrenos. Hoy gracias al poco éxito que, 
en la mayor parte de las ocasiones, tuvieron esos polígonos industriales, muchos de esos espacios han vuelto a reutilizarse para la agricultura.

En resumen, el panorama anterior a la localización de la factoría de General Motors en Figueruelas es el de una extensión muy débil de este "Corredor Industrial" en forma radial que desde su núcleo origen, Zaragoza, había ido difundiendo su influencia a través de los ejes de entrada a la ciudad y en donde todavía había una fuerte pervivencia de las instalaciones industriales pequeñas y medianas en el interior de su casco urbano. Fuera de este espacio lo que encontramos era una localización muy puntual en núcleos semiurbanos de la provincia (Ejea de los Caballeros, Calatayud,...) donde los polígonos de desarrollo habían conseguido captar alguna inversión.

\section{IMPLANTACION DE GENERAL MOTORS Y LOS CAMBIOS EN EL MODELO DE LOCALIZACION INDUSTRIAL.}

Creemos que es necesario marcar una tercera etapa en los modelos de localizaciones industriales en Zaragoza a partir de 1981 con la llegada de General Motors E., por la importancia que esta empresa tiene para Aragón y más concretamente para Zaragoza. Esta empresa ha traido consigo un nuevo gusto por las ubicaciones industriales en donde las distancias kilométricas apenas importan, si las infraestructuras de comunicaciones son buenas.

La factoría de Figueruelas (Vid. croquis de situación) constituye un caso totalmente innovador de asentamiento industrial en Zaragoza, tanto por su tamaño (en superficie y en número de empleados) como por la elección del lugar concreto de ubicación (bastante distante de un centro urbano, a $30 \mathrm{Km}$.) y por el tipo de instalación (con utilización de una tecnología muy avanzada).

La crisis que el sector industrial sufrió en nuestro país durante los años setenta fue superada tempranamente en la región aragonesa gracias a la instalación, a comienzo de los ochenta, de la factoría de automóviles que General Motors montó en Figueruelas. La fuerte inversión que esta sola empresa realizó, superaba en muchos puntos la acometida en los años anteriores dentro de esta región. 
La planta de esta Multinacional tendrá influencia no sólo por las repercusiones producidas por su localización concreta, sino por la atracción e imitación que está causando sobre otras empresas industriales, que se van alejando cada vez más de sus anteriores localizaciones en el centro urbano de la ciudad, fuente principal de su mano de obra (Vid. mapa de distribución del número de empleados de General Motors en 1989). Esto es posible gracias al importante desarrollo de los transportes por carretera y por la mejora de las infraestructuras. Además hoy la mayoría de instalaciones industriales se ven obligadas a aplicar tecnologías más avanzadas para poderse equiparar con el resto de empresas, ello obliga, a su vez, a necesitar de amplios espacios de terreno que sólo se encuentran a distancia del centro de la ciudad.

La importancia que esta sola empresa tiene, ha permitido la extensión del área de influencia de la propia ciudad de Zaragoza, puesto que es de ella de donde mayores aportes de trabajadores recibe la factoría y, por tanto, hoy la primera banda de influencia de Zaragoza no acaba en Utebo, ni en Casetas sino que se prolonga en torno a Figueruelas. A todo este espacio los cambios acaecidos lo van acercando, cada vez más, hacia pautas de tipo urbano.

\section{CONSOLIDACION DEL "CORREDOR INDUSTRIAL DEL EBRO"}

La consolidación de este corredor se ha configurado a través de la expansión de las localizaciones industriales en los distintos ejes de entrada a la ciudad de Zaragoza, donde la densidad y la distancia al centro urbano van siendo mayores. Hasta hace poco tiempo se podían apreciar en todos los ejes de entrada importantes huecos entre unas instalaciones y otras, hoy poco a poco estos vacíos se van completando y empiezan a constituir un continuo de empresas en las márgenes de las distintas carreteras que confluyen en la ciudad.

Las previsiones del Plan Yarza de finales de los años cincuenta (1957), para la expansión de la ciudad por sus alrededores han quedado completamente desbordadas. El crecimiento industrial ha rebasado todas los perspectivas imaginables, dando lugar a una periferia mucho más extensa a causa de la gran proliferación industrial que en la década de los ochenta se ha producido. 
Las zonas exteriores de la ciudad se han desarrollado enormemente, pero lo han hecho de forma totalmente aleatoria. Durante mucho tiempo no han tenido demasiada cohesión unos establecimientos con otros, permaneciendo aislados, tampoco ha existido mucha relación entre el tipo de sector industrial que se ubicaba en una zona u otra, ni se han tenido en cuenta los espacios utilizables más rentablemente para la agricultura que para el uso industrial, puntos todos ellos sobre los que no se había planificado al no pensar en el enorme desarrollo que ha alcanzado el dinamismo industrial.

Desde 1960 hasta 1984 ha sido constante la instalación de industrias en las áreas externas de la ciudad. Pero hay dos momentos claves para el asentamiento de estas instalaciones en el exterior del casco urbano de Zaragoza: el primero coincide con la aprobación de Zaragoza como Polo de Desarrollo (1964-69) y el segundo con la fecha de instalación de General Motors (1980-84).

Este último hecho es lo que nos ha dado pie para poder considerar una nueva etapa y modelo en las localizaciones industriales a partir de esa instalación. Esta empresa tanto directa como indirectamente está dando un fuerte impulso a nuevos modelos de concebir las zonas para instalar las industrias, donde la distancia kilométrica no importa si lo que se requiere es una buena salida del producto y amplios espacios para las distintas naves.

La implantación de esta factoría ha traido consigo un refuerzo en el tipo de localización industrial, cada vez más alejado de los centros urbanos y la consolidación en torno a ella del "Corredor Industrial del Ebro", como un espacio no sólo de paso sino también de ubicación industrial a lo largo de las vías de comunicación.

Con su presencia, esta Multinacional está ejerciendo un efecto imán sobre otras pequeñas empresas que pretenden vivir directa o indirectamente del "gran gigante", instalándose en sus proximidades y aprovechando las propias infraestructuras que esta gran empresa utiliza. A su vez, otras empresas tienden a imitar este modelo de localización asentandose en distintas zonas más externas del centro urbano, junto a buenas vías de comunicación que les permitan accesos fáciles a cualquier punto de la ciudad y a otras zonas nacionales o extranjeras con las que tengan que relacionarse. 
En este modelo el eje de la Autovía de Logroño ha sido el primero en alargar su extensión superficial en la implantación industrial, por el "efecto imán" que ha producido General Motors, por lo que este tramo se está convirtiendo hoy en uno de los más dinámicos de este Corredor.

El aspecto que muestran hoy el resto de localizaciones industriales tampoco es el mismo que el encontrado por General Motors en los primeros momentos de su instalación. Existen todavía pequeñas industrias en el interior del propio casco urbano, vestigios de la formación de este corredor, en las cuales, su propia dimensión nos demuestra que difícilmente pueden tener buenos beneficios si no se adaptan al nuevo modelo de implantación espacial y a las nuevas tecnologías que se están aplicando ya en muchas otras empresas asentadas en el exterior de la ciudad. Los problemas de tráfico, la falta de espacios con que poder aumentar sus instalaciones, es lo que a la larga les influirá para trasladarse si quieren seguir avanzando en el mundo industrial y no quedarse como meros artesanos.

Al encontrarse saturadas las zonas más próximas al casco urbano, las industrias tienden a alejarse cada vez más del centro urbano, expandiéndose a través de los distintos ejes de comunicación, especialmente el de la Autovía de Logroño y el de la Carretera de Barcelona, tanto por la relevancia de las instalaciones que en ellas se ubican ya como por ser los ejes que comunican directamente con otras zonas nacionales económicamente más importantes.

El aspecto general que hoy presentan cada uno de los ejes para su su posible expansión es el siguiente:

- Carretera de Huesca, el tipo de industrias situadas en ella no son de gran tamaño, puesto que al estar ocupada en gran parte por un espacio militar, le resta posibilidades de crear una zona industrial importante, hay que pensar además que esta carretera no constituye ningún eje de comunicación principal con otras zonas nacionales industrializadas, como les sucede al resto de los demás ejes que penetran en la ciudad, pero la próxima construcción de la autovía con Huesca y la apertura del paso de Canfranc puede incrementar las instalaciones en este espacio. 
- Carretera de Logroño es el eje que mayor dinamismo ha tenido en cuanto a la localización de industrias en él, puesto que ya no es solamente General Motors, sino todas las pequeñas industrias que en sus proximidades están surgiendo ${ }^{7}$. Es la zona industrial más extensa, aunque no todo ese eje hasta Figueruelas está ocupado. Sin embargo, se aprecia como la densificación es cada día más alta.

Además de General Motors otras dos grandes empresas que se asientan también en este eje son Pikolín y Valeo Térmico, ambas con plantillas de trabajadores bastante importantes, pero a mucha distancia de los que tiene la Multinacional.

Hay que destacar, también de esta carretera, su importante desarrollo comercial, tanto de ventas al por mayor en grandes almacenes como de ventas al por menor en hipermercados y tiendas que para sus exposiciones necesitan de amplias superficies, como les sucede a las tiendas de muebles, muy numerosas en este corredor. Estas instalaciones atraidas por la fuerte circulación de personas sobre este eje están contribuyendo a una densificación mucho mayor sobre esta vía.

- Carretera de Madrid es un eje bastante débil en cuanto a instalaciones industriales. Estas son de tamaño medio, aunque gracias a la construcción de la autovía con Madrid está conociendo un importante crecimiento en estos últimos años. La empresa de mayor tamaño es la Van Hool (Hispano Carrocera) ${ }^{8}$.

- Carretera de Valencia, sigue estando dominado por la presencia de numerosas industrias de pequeño tamaño, con plantillas de empleados muy reducidas. Su presencia ha transformado la fisonomía de los municipios de este eje, especialmente en Cuarte de Huerva.

7 En las inmediaciones de General Motors, los municipios más próximos están acondicionando importantes superficies de terreno industrial (Figueruelas, Pedrola, Alagón, Pinseque, Epila, Borja,...), donde ya se están instalando muchas de las empresas proveedoras de la Multinacional. Este crecimiento industrial está ocasionado por la nueva política de compras de esta empresa basada en el sistema "just in time", por el que ésta pretende reducir los gastos y los inconvenientos del transporte de los materiales de montaje que necesita. Por ésto sus proveedores se ven obligados a instalarse a menos de trenta kilómetros de la factoría automovilística, potenciando el desarrollo industrial de su espacio inmediato.

8 Hispano Carrocera ha adquirido unos terrenos para trasladarse a la Carretera de Castellón, en la Cartuja. En los que ocupa hoy van a construirse bloques de viviendas. 
- Carretera de Castellón la continuidad de este eje es quizá mayor que en otros. Sin embargo, es el que menos espacio tenía para la ocupación industrial por lo que en gran parte se han movido a través de instalaciones ilegales.

Carretera de Barcelona es también un eje donde las densidades industriales son altas a causa de la concentración industrial hasta aproximadamente unos 13 kilómetros desde la salida en la Avenida de Cataluña. En esta zona resulta difícil encontrar espacios sin ocupar, entre las empresas mayores que están allí ubicadas, destacan: Balay, la Compañía Auxiliar de Ferrocarril (CAF), Nurel,.... Después de terminar esta primera concentración, se ubica el polígono de Malpica donde durante mucho tiempo las parcelas han estado vacías aunque hoy la progresiva ocupación es un hecho.

- El eje del Jalón, que hasta ahora salvo en momentos muy concretos, como en la etapa de desarrollo de las azucareras, había permanecido al margen de la expansión industrial del eje del Ebro, hoy debe ser incluido dentro de la delimitación de éste porque es una de las principales zonas hacia las que se está dirigiendo el influjo de General Motors, tanto de forma directa a través de los trabajadores que desde distintos municipios de esta zona se trasladan hasta la factoría, como indirectamente a través del efecto imán que sobre otras empresas industriales está ocasionando, especialmente sobre sus empresas auxiliares que por la nueva política de aprovisionamiento de General Motors ("just in time") se ven atraidas hacia este eje para su instalación ${ }^{9}$.

El elemento común en la industrialización de todo este espacio ha sido la falta de una coordinación en su desarrollo, las distintas empresas fueron surgiendo de forma aislada, dejando importantes espacios entre ellas y que hoy poco a poco con la importante demanda de suelo industrial, que durante la década de los ochenta se ha producido, esos espacios se están completando.

\footnotetext{
${ }^{9}$ La carretera comarcal de ribera del Jalón, en la que se ha prolongado la autovía hasta la entrada a la planta de General Motors, está siendo objeto de una importante mejora añadiendo arcenes y ensanchando la calzada, porque las condiciones que presentaba eran muy poco favorables para una buena comunicación que puede unir la factoría con la Autovía de Madrid. En breve plazo de tiempo esta vía puede ser uno de los puntos que potencie el desarrollo de la ribera del Jalón.
} 
Pero cuando nos referimos en este artículo al "Corredor Industrial del Ebro", no sólo lo hacemos pensando en la ciudad de Zaragoza y su efecto de expansión por su periferia, sino en un espacio mucho más amplio que poco a poco, partiendo del desarrollo de esta ciudad se ha ido alargando en su crecimiento por toda la comarca de la Ribera del Ebro (desde Novillas hasta Caspe), donde encontramos una serie de núcleos que están adquiriendo o recobrando un gran dinamismo (Fuentes de Ebro, Utebo, Pinseque, Alagón, Figueruelas, Pedrola, Gallur,...).

En esta delimitación habría que incluir las zonas de confluencia entre el río Ebro y sus afluentes principales, como el río Huerva hasta el municipio de María de Huerva, o el río Gállego hasta Villanueva de Gállego y en especial el río Jalón con la ubicación de factoría de General Motors, en un lugar cercano a la confluencia entre el río Ebro y el Jalón, motivo por el que se está beneficiando de la expansión del "Corredor Industrial del Ebro" 10 .

Por último, dentro de este mismo dinamismo se pueden englobar otra serie de enclaves más puntuales y alejados de to que se conoce como eje del Ebro: Ejea de los Caballeros, Tarazona, Tauste, Borja, Calatayud, La Almunia de Da Godina o Epila, núcleos ubicados sobre importantes carreteras nacionales y donde se localizan una serie de polígonos industriales, cuya situación actual los acerca al dinamismo de este "Corredor" y los separa del desarrollo de sus propias comarcas.

\section{RAZONES DE CRECIMIENTO DEL "CORREDOR INDUSTRIAL DEL EBRO"}

Las principales razones de consolidación de este corredor coinciden con las que General Motors buscó para la ubicación:

- A nivel nacional, este espacio se encuentra entre las zonas económicamente más desarrolladas de España. Concretamente Zaragoza constituye el centro del llamado "Cuadrante Fértil Peninsular", es el paso obligado de las comunicaciones entre las ciudades españolas más importantes (Madrid-Barcelona, Barcelona-País Vasco, Valencia-

10 Es de destacar como la representación cartográfica de este espació coincide claramente con el área de atracción de obreros que tiene General Motors. 
País Vasco, Valladolid-Barcelona). Con todas ellas posee hoy unas comunicaciones terrestres muy aceptables 11 .

- A nivel internacional, este eje es también paso obligado de comunicaciones. Está cerca y bien comunicado con la frontera francesa tanto por Irún como por Port Bou 12

Sin embargo, a estas características que aparecen en todo el eje del Ebro hay que añadir algunas más específicas que durante los últimos años han ido conformando dentro de todo este eje un espacio más concreto, que se ha pasado a denominar "Corredor Industrial del Ebro" donde se está produciendo una fuerte expansión industrial. La zona que delimita este corredor posee los siguientes elementos que la potencian:

- Buenos accesos: por carreteras, ferrocarril o por avión. Tanto en carreteras nacionales como por las autovías que parten desde Zaragoza hasta distintas poblaciones cercanas a ella, en cuyas márgenes se localizan las zonas industriales más consolidadas, o por autopistas, el "Corredor" se encuentra muy bien comunicado con los princiapales centros nacionales y también extranjeros. Por ferrocarril, constituye también nudo importante de contacto con muchas ciudades españolas y del exterior (Zaragoza-Madrid, Zaragoza-País Vasco, Zaragoza-Barcelona, Zaragoza-Valencia), a lo que hay que añadirle que Zaragoza es también una de las ciudades por donde se proyecta el paso del tren del alta velocidad y la reapertura de la línea de Canfranc. Zaragoza posee aeropuerto que puede ser utilizado tanto para el tráfico de pasajeros como de mercancías

- Rapidez de comunicación con los mercados europeos y peninsulares. La buena accesibilidad desde este espacio a las fronteras europeas, donde con la próxima apertura del mercado

11 A través de carreteras nacionales Zaragoza se encuentra unida con Vitoria, Bilbas, Logroño, Huesca, Castellón, Valencia, Teruel, Lerida, Tarragona, etc.,..

- Con autovía hasta María de Huerva (carretera de Valencia), Pedrola (carretera de Logroño), Villanueva de Gallego (carretera de Huesca) y Madrid (sin terminar).

- Con autopista hasta Valencia, Castellón, Tarragona, Lérida, Barcelona, Burgos, Logroño, Pamplona, Vitoria y Bilbao.

12 Está aprobada la realización del proyecto de apertura de un tunel por Somport. Este tunel, cuya finalización se prevee para finales de 1995, permitirá una rápida comunicación del corredor del Ebro con Pau y a su vez con el resto de enclaves europeos, sin tener que desplazarse hasta Irún o la Junquera para atravesar la frontera. 
único europeo se abren buenas expectativas para las producciones industriales. A través tanto de Irún por la Autopista del Cantábrico como de Port Bou por la Autopista del Mediterráneo, se puede acceder fácilmente desde este lugar a la frontera francesa. A esto se le pueden sumar los medios de transmisión ${ }^{13}$ que hoy posee y que van a ser ampliados en breve cuando se termine la Torre de Comunicaciones de Zaragoza.

- Población suficiente para cubrir un número importante de puestos de trabajo. La ciudad de Zaragoza había aumentado extraordinariamente su población, poseía una gran cantidad de población activa con una fuerte tradición en el trabajo de talleres y tenía todos los servicios sociales e infraestructuras que la misma requería.

- Importante capacidad de suministros de agua (proximidad del Ebro, Jalón y del Canal Imperial), facilidad de vertidos, abastecimientos de terrenos, de electricidad ${ }^{14}$ (pasan importantes líneas de alta tensión que permiten cubrir bien la demanda), de gas natural 15 , de hidrocarburos (con facilidad de acceso a redes de transporte) etc.,...

A estas ventajas, se pueden añadir aspectos como la escasa conflictividad que, en general, muestran las plantillas de las empresas de este espacio, la disponibilidad a la financiación que muchos organismos ofrecen o las facilidades dadas por las distintas administraciones locales, provinciales o autonómicas. Estas canalizan toda una serie de ayudas para que las distintas empresas que buscan o han buscado un lugar para su ubicación lo hagan en en este espacio. Estas ayudas van dirigidas a: la adquisición de los terrenos, la preparación de las infraestructuras de comunicaciones y equipamientos, suministros de agua, de energía, ordenación urbanística de la zona, etc,...

\footnotetext{
13 Sistema de transmisión por cable óptico con Barcelona, Lérida, Pamplona, Guadalajara, Madrid y Alcañiz. Con cable coaxial con Barcelona, Bilbao y Madrid.

14 Si tomaramos la petición de la ampliación de kilowatios industriales como punto de apoyo para la delimitación de la zona de mayor expansión industrial en la provincia de Zaragoza en los últimos diez años obtenemos que las principales ampliaciones en la red se localizan en los núcleos de este corredor industrial: Pinseque, La Joyosa, Sobradiel, San Mateo de Gállego, Villanueva de Gállego, Alfajarín, El Burgo, María de Huerva, La Muela, Epila, Figueruelas y Pedrola.

15 Paralela a este corredor industrial existe una red de transporte de gas natural que es aprovechada, a través de distintas arterias de salida, por las principales zonas industriales de este espacio, entre ellas por General Motors que cuenta con una arteria propia de abastecimiento, pero que en breve va a poder ser utilizada por las empresas que se asienten en los polígonos industriales que se están consolidando en sus inmediaciones.
} 
Todas estas condiciones favorables fueron las aprovechadas en su día por General Motors y hoy lo están siendo por muchas otras empresas que atraidas por este " gran gigante" se instalan en sus inmediaciones, ayudando al crecimiento del "Corredor Industrial del Ebro".

\section{CONCLUSIONES}

Todo este espacio que a grandes rasgos acabamos de definir como el "Corredor Industrial del Ebro" es muy amplio y no ha terminado de configurarse al estar en una etapa de expansión. Aunque no se puede olvidar que si bien aparece ya consolidado en torno a los ejes de entrada a la ciudad de Zaragoza, no sucede lo mismo en el resto de enclaves con un desarrollo todavía muy puntual y sobre los que se pretende inyectar el mismo tipo de dinamismo que el infundido sobre el espacio ya consolidado. Por ello nuestro análisis sobre este tema apunta hacia la delimitación señalada, pero queremos señalar que no nos ofrecen una demarcación todavía demasiado clara

Una de las principales conclusiones que se pueden extraer de este estudio es que definitivamente lo que parecía que ya se había configurado tan sólo como una "zona de paso" entre las áreas más industrializadas del país, característica ofrecida gracias a que se había dotado a este espacio de buenas infraestructuras, no se ha cumplido; ya que por fin el valle medio del Ebro se está consolidando como uno de los enclaves más dinámicos del País, sobre el que se están asentando numerosas instalaciones industriales. Aunque este hecho no termina con los fuertes desequilibrios que sufre la región aragonesa, al menos extiende su desarrollo sobre un espacio mucho más amplio a lo largo del eje del Ebro e incluso lo infunde a otros espacios próximos como es el caso del valle del Jalón, espacio que se está potenciando a partir del propio desarrollo del "Corredor del Ebro".

Por último, reseñar que si hay que buscar un responsable de la consolidación de este "Corredor" hay que acudir a la presencia de General Motors E.. Esta empresa que por sí sola está produciendo gran parte de los hechos indicados y causando uno de los impactos espaciales más importantes de la región por el efecto de atracción de otras industrias y servicios sobre este eje. Además ha terminado con el tópico de Aragón "región de paso", ya que son muchas las empresas 
que después de la instalación de esta Multinacional han decidido también imitándola asentarse en el espacio del "Corredor".

\section{BIBLIOGRAFIA}

ALEGRE, L. y Otros (1991): " La empresa industrial (1984-1989)". Papeles de Economía Española, Comunidades Autónomas, $\mathrm{n}^{\circ} 10$ Aragón.

ALONSO LOGROÑO, M.P.(1991): "Impacto de General Motors E. Estudio del Medio Rural Circundante". Memoria de Licenciatura. Inédita.

BAGUENA SANCHEZ, J.A. (1991): " La economía en eje del Ebro". Papeles de Economía Española, Comunidades Autónomas, $n^{9} 10$ Aragón.

BIESCAS FERRER, J. (1978): "El Proceso de industrialización en el Aragón contemporáneo" J.E.A.E.S.A. Zaragoza.

BIESCAS FERRER, J.A. (1991): "Los diferentes espacios económicos aragoneses". Papeles de Economía Española, Comunidades Autónomas, nº 10 Aragón.

FRUTOS MEJIAS, L. M ${ }^{a}$ y SOLANS, M. (1975): "El impacto del desarrollo industrial en la morfología urbana de Zaragoza".Ciudad e Industria, IV Coloquio de Geografía, A.G.E.. Oviedo.

FUNDACION NUEVA EMPRESA (1991):"Estudio para la implantación de empresas en el Corredor Industrial del Ebro". 


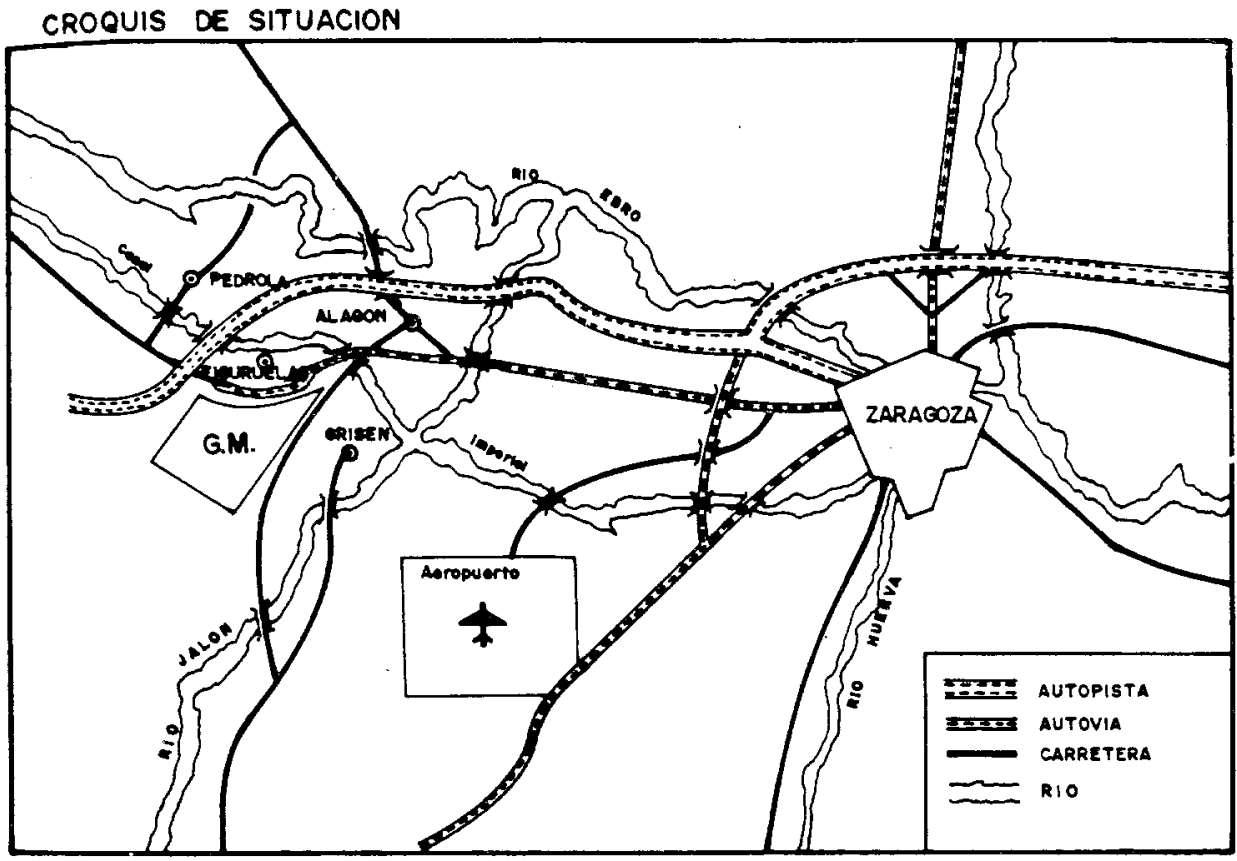

FUENTE : GENERAL MOTORS 


\section{DISTRIBUCION DEL NUMERO DE EMPLEADOS}

DE GENERAL MOTORS EN 1989

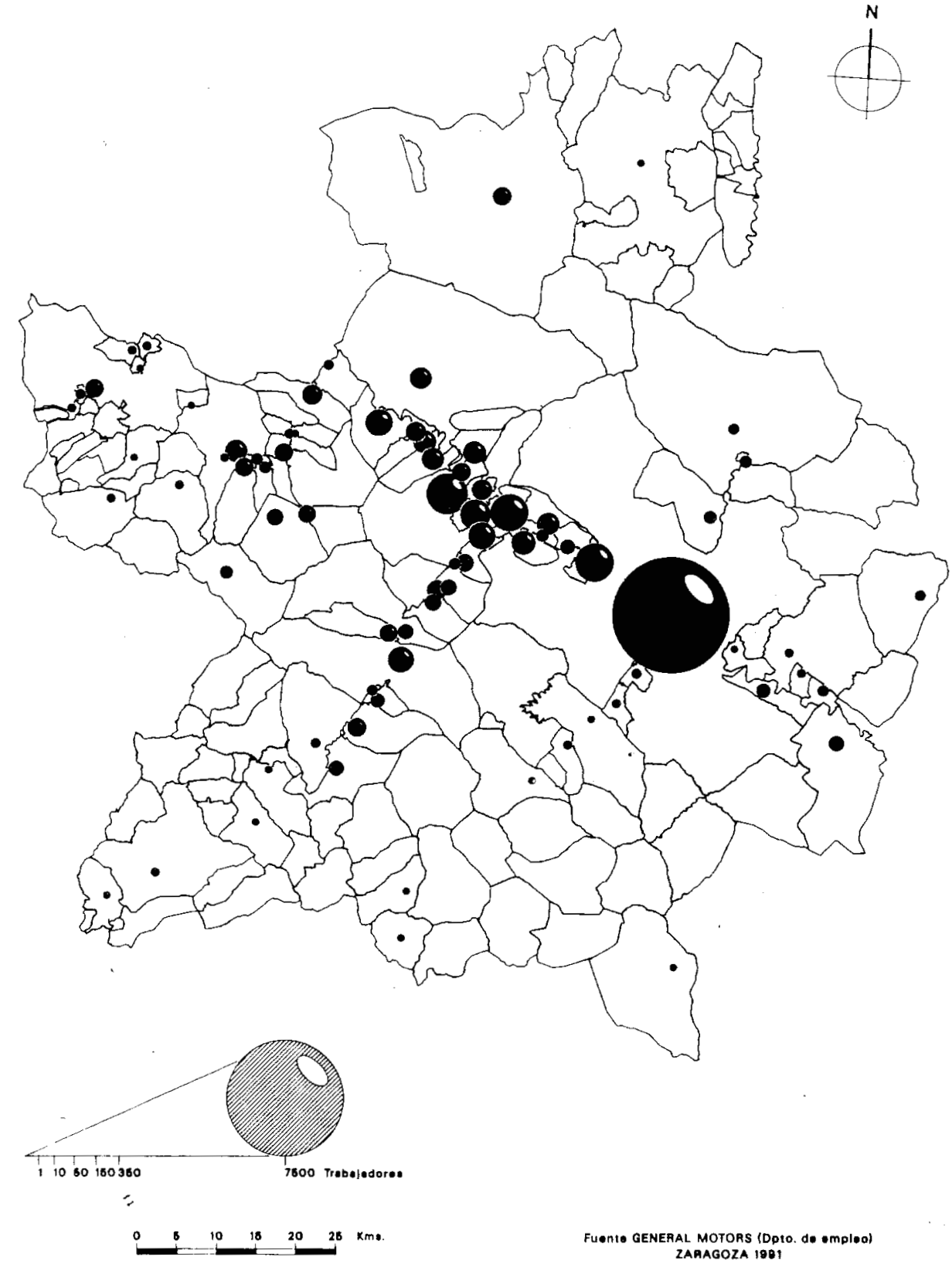

